\title{
Reliable Estimation of Heart Surface Motion under Stochastic and Unknown but Bounded Systematic Uncertainties
}

\author{
Evgeniya Bogatyrenko, Benjamin Noack, and Uwe D. Hanebeck
}

\begin{abstract}
A reliable estimation of heart surface motion is an important prerequisite for the synchronization of surgical instruments in robotic beating heart surgery. In general, only an imprecise description of the heart dynamics and measurement systems is available. This means that the estimation of heart motion is corrupted by stochastic and systematic uncertainties. Without consideration of these uncertainties, the obtained results will be inaccurate and a safe robotic operation cannot be guaranteed. Until now, existing approaches for estimating the motion of the heart surface are either deterministic or treat only stochastic uncertainties. The proposed method extends the heart motion estimation to the simultaneous consideration of stochastic and unknown but bounded systematic uncertainties. It computes dynamic bounds in order to provide the surgeon with a guidance by constraining the motion of the surgical instruments and thereby protecting sensitive tissue.
\end{abstract}

\section{INTRODUCTION}

Beating heart operations are still a challenging task for surgeons. They pose high demands on the synchronization of surgical instruments with the heart surface motion. A robotic surgery system can overcome the limitations of human abilities by providing fine manipulation capabilities and improving the reliability. However, it has to meet high requirements on the robustness and accuracy of the estimation and tracking algorithms.

According to [1], an appropriate accuracy should be about $0.1 \mathrm{~mm}$ in order to allow for operations on coronary arteries of diameter $0.5 \mathrm{~mm}-2 \mathrm{~mm}$. Nevertheless, existing tracking approaches can only achieve an average accuracy of $1 \mathrm{~mm}-$ $1.5 \mathrm{~mm}[2]$. Such a low accuracy affects the safety of robotic operations. Since active medical robots may directly contact the patient for penetrating, cutting, and removing heart tissue, safety is one of the key issues.

One of the reasons for the degradation of the accuracy, and hence the safety, is that the model used for estimating the heart surface motion and the measurements are corrupted by disturbances. However, in the majority of the methods for compensating the heart surface motion, these uncertainties are not considered [3]-[6].

In general, there are two types of uncertainties that lead to errors in the estimation process. Stochastic uncertainties can be described statistically and their effect can be compensated by repeated measurements. In process dynamics, uncertainties are caused by input noise. The observations, provided by

E. Bogatyrenko, B. Noack, and U. D. Hanebeck are with the Intelligent Sensor-Actuator-Systems Laboratory (ISAS), Institute for Anthropomatics, Karlsruhe Institute of Technology (KIT), Karlsruhe, Germany. bogatyrenko@kit.edu, noack@kit.edu, uwe.hanebeck@ieee.org a camera system, are affected by stochastic uncertainties arising from electronic noise, flickering, imprecision of feature extraction, or distortion [7]. In contrast to stochastic errors, systematic uncertainties cannot be analyzed statistically and are difficult to detect, especially when they are not constant over time. In process dynamics, these uncertainties arise from an inaccurate estimation of model parameters, imprecision in model geometry, linearization and approximation errors, a bias of the input signal, or inadequate modeling. The systematic uncertainties of the observations are associated with the limited validity of the measurement system model due to either calibration inaccuracies or nonlinearities that this calibration could not take care of.

Some existing approaches for estimating heart surface motion can cope with stochastic uncertainties [8]-[11]. A common way is to apply the Bayesian inference scheme. For linear systems affected by Gaussian noise, the Kalman filter [12] is the optimal estimator. In nonlinear cases, nonlinear estimation procedures, e.g., the extended Kalman filter [12], are employed. A Bayesian inference scheme assumes that the state is characterized by a unique probability density. This can only be maintained if an accurate model of the process dynamics and observations is available. When the system is affected by unknown stochastic or systematic disturbances, large estimation errors can occur due to incorrect modeling [13]. Therefore, neglecting unknown disturbances implies a lack of precision that greatly increases the risk of complications during beating heart robotic surgery. Until now, systematic uncertainties have not been considered in the estimation of heart surface motion.

Therefore, we will extend the estimation of heart surface motion to the simultaneous consideration of stochastic and systematic uncertainties, in order to achieve robust and safe robotic operations. Our approach aims at being integrated in the two most common safety concepts for robotic surgery: safety critical limits [14] and virtual fixtures [15].

The key idea of safety critical limits is to distinguish between safe and unsafe operating conditions. Inside the defined limits, the controller must be designed to prevent, eliminate or reduce the possibility of a robot safety hazard to an acceptable level. The other method to increase the safety of robotic operations is to define virtual fixtures that constrain the robot motion to a predefined region, such as the heart surface. These fixtures still permit surgeons to retain ultimate control, but extend their capabilities, enhance the speed of surgery, and reduce mental stress by restricting robot motion [16]. For cardiac surgery, the concept of virtual fixtures has first been proposed in [15]. It is extended to beating 

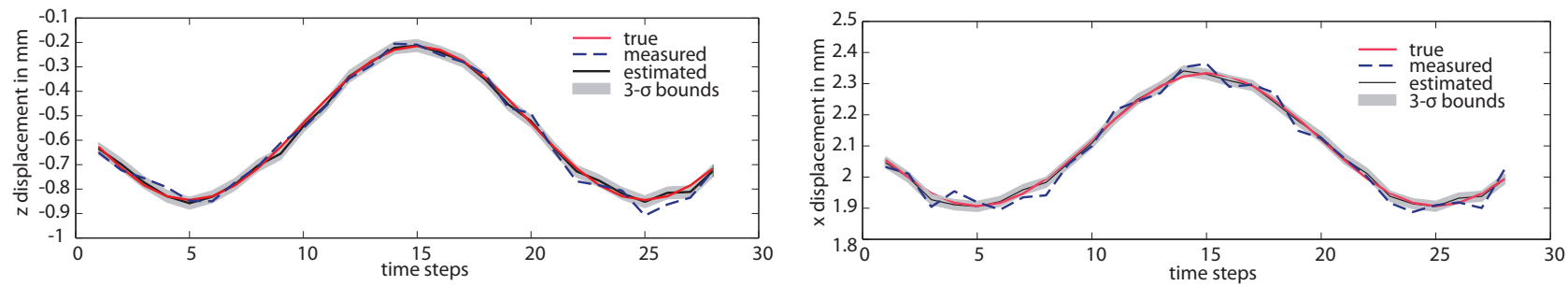

(a) State estimation based on process dynamics and observations corrupted by stochastic errors with known distributions. The true state is inside the $3 \sigma$ bounds.
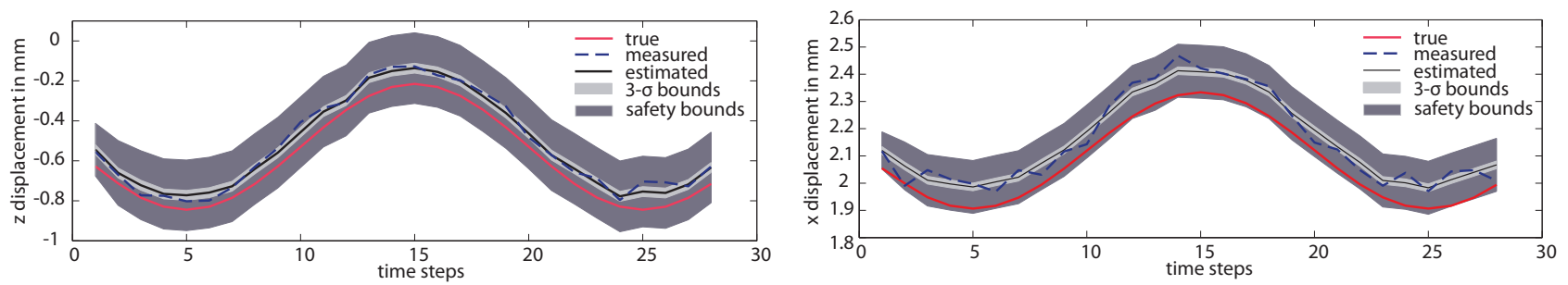

(b) State estimation based on process dynamics and observations corrupted by stochastic and unknown but bounded systematic errors. The true state is outside the $3 \sigma$ bounds.

Fig. 1. Appropriate state estimation is only guaranteed when accurate models for process dynamics and observations are available. If the system is affected by systematic disturbances, a purely stochastic description of a system implies a lack of precision.

heart procedures in [17]. Commonly, safety critical limits and fixtures are static bounds generated from preoperative procedures. A performance of the static bounds degrades in cases of occlusions or incorrect models.

In this paper, we propose the concept of dynamic safety bounds that enable us to cope with the aforementioned problems. They can be used for matching the robot motion to the heart surface motion. The computation of these bounds is based on the robust estimation of the heart surface motion considering stochastic and unknown but bounded systematic uncertainties in the process dynamics and observations. Finally, these bounds allow for the instantaneous evaluation of estimation quality, so that soft tissue can be protected in case of inaccuracies.

\section{SAfety in Robotic-Assisted Applications}

Before the mathematical background is explained, this section will highlight the necessity for simultaneously considering stochastic and systematic errors. In this way, the robustness and reliability of robot-assisted applications, such as beating heart operations, can be improved significantly. After motivating the combined treatment of stochastic and systematic errors, we will propose the calculation of safety bounds for constrained motion of robotic instruments.

In order to point out the impact of systematic errors, we consider the example of estimating the $x$ - and $z$-displacement of a beating heart from noisy measurements. The heart motion and measurements are simulated. We assume that an accurate model of the process dynamics and observations is available and that the measurements are only corrupted by zero-mean white Gaussian noise with variance $\sigma^{2}=$ $0.001 \mathrm{~mm}^{2}$. A Bayesian estimator can then compute state estimates through recursive prediction and filtering. In the example depicted in Fig. 1(a), the true heart displacement lies within the $3 \sigma$ confidence limits around the estimated means.
The state estimates presented in Fig. 1(b) are calculated from measurements that are additionally affected by a systematic bias of $0.1 \mathrm{~mm}$. The true state now lies outside the $3 \sigma$ bounds. Since the systematic uncertainties are unknown and cannot be estimated by conventional estimation approaches, there is no knowledge about the position of the true state available. Thus, unconsidered systematic disturbances can result in unreliable robotic assistance. The same pertains to stochastic uncertainties with unknown distributions. In the considered example, the surgical instruments might penetrate the surface of the heart too deeply or too shallowly due to the inconsistent estimates of its $z$-displacement. The coronary arteries near an intervention area can also be injured due to the inaccurate estimation of the $x$-displacement.

In order to cope with unknown systematic errors, a common way is to determine bounds for these errors. In the considered example, the bias on the measurements is assumed to be unknown but bounded by $\pm 0.12 \mathrm{~mm}$. This bound can now be converted into bounds for the system estimate, which can then be propagated over time and updated by further measurements. More precisely, these bounds characterize a set of possible state estimates at each time step. By extending the lower and upper boundary by the $3 \sigma$ confidence limits, we obtain certain safety bounds for the true system state, which are represented by the shaded region in Fig. 1(b). This ensures that the true system state is inside this region with a probability of at least $99.73 \%$.

For the purpose of handling stochastic and systematic uncertainties, two main challenges have to be addressed:

1) Stochastic and systematic uncertainties have to be identified and distinguished in the system and observation models and have to be characterized appropriately. Against the background of beating heart operations, this will be explained in Section III and IV. 
2) The system state has to be reconstructed from measurements affected by stochastic and systematic errors. For this, a combination of Bayesian and set-valued state estimation will be employed by utilizing sets of densities. Section V will explicate how to propagate and update the error bounds for the state estimates.

The computed safety bounds can now be used for haptic guidance, as proposed in [17]. They can represent dynamic soft virtual fixtures, in order to prevent the robot from accidentally harming healthy tissue. These features can help an operator or robot to move along the heart surface, with the operator having the option to reject the haptic guidance by applying additional force. More precisely, the surgeon should feel constraint forces when the surgical instrument is just about to move inside the boundary of the desired zone. The velocity and degree of freedom of robotic instruments should be constrained in this area. In order to guarantee the safety of the robotic operation when penetrating, cutting or removing heart tissue in this zone, an additional measurement system such as force sensor should be used. A force sensor will increase the measurement accuracy only in $z$-direction. In $x$ - and $y$-direction, the motion of robotic instruments inside the safety bounds should be strongly constrained. In contrast to static bounds, the advantages of the proposed dynamic error bounds are that they reflect the measurement system's accuracy at every time instant, make the quality of the estimates assessable, and ensure with a certain probability that the true heart surface is inside these bounds.

\section{STATE-SPACE MOdEL}

Common approaches to state estimation require a system description in state-space form. For that purpose, the heart surface motion should be described by system and measurement equations that represent the process dynamics and observation models.

By assuming that the heart surface behaves like a linear elastic physical body with isotropic material structure, the heart dynamics can approximately be characterized by a deterministic discrete-time linear system, according to [8], [10], [18]. These assumptions are valid if a small intervention area of the heart surface is considered and the heart motion is constrained by a mechanical stabilizer.

The model of the process dynamics, or the system equation, relates the states of the heart movement at different time steps. Since the model is affected by stochastic noise $\underline{\boldsymbol{w}}_{k}$ and systematic perturbations $\underline{d}_{k} \in \mathbb{R}^{n}$, the predicted state $\underline{\boldsymbol{x}}_{k+1} \in \mathbb{R}^{n}$ can be described by

$$
\underline{\boldsymbol{x}}_{k+1}=\mathbf{A}_{k} \underline{\boldsymbol{x}}_{k}+\mathbf{B}_{k}\left(\underline{\hat{u}}_{k}+\underline{\boldsymbol{w}}_{k}\right)+\underline{d}_{k},
$$

where $\mathbf{A}_{k} \in \mathbb{R}^{n \times n}$ represents the system matrix, $\mathbf{B}_{k} \in$ $\mathbb{R}^{n \times m}$ denotes the input matrix, and the state vector $\underline{\boldsymbol{x}}_{k+1}$ describes the distributed heart surface displacement caused by a known input $\underline{\hat{u}}_{k} \in \mathbb{R}^{m}$. This input is determined by the atrial pressure that can be monitored in cardiac surgery by a central line catheter [19]. The error term $\underline{\boldsymbol{w}}_{k} \sim \mathcal{N}\left(\underline{0}, \mathbf{C}_{k}^{w}\right)$ is a zero-mean white Gaussian noise with covariance matrix $\mathbf{C}_{k}^{w} \in \mathbb{R}^{n \times n}$.

Given that the heart surface position is reconstructed from the measurements provided by a camera system, the observation model can approximately be assumed to be linear. This model is characterized by the measurement equation that relates the obtained observation $\hat{y}_{k} \in \mathbb{R}^{l}$ at time step $k$ to the state $\underline{x}_{k} \in \mathbb{R}^{n}$. By considering the stochastic noise $\underline{\boldsymbol{v}}_{k}$ and systematic uncertainties $\underline{e}_{k} \in \mathbb{R}^{l}$, the observation model can be written in the form

$$
\underline{\hat{y}}_{k}=\mathbf{H}_{k} \underline{\boldsymbol{x}}_{k}+\underline{\boldsymbol{v}}_{k}+\underline{e}_{k},
$$

where $\mathbf{H}_{k} \in \mathbb{R}^{l \times n}$ represents the measurement matrix and $\underline{\boldsymbol{v}} \sim \mathcal{N}\left(0, \mathbf{C}_{k}^{v}\right)$ is a white zero-mean Gaussian noise with covariance matrix $\mathbf{C}_{k}^{v} \in \mathbb{R}^{l \times l}$. The vector $\underline{e}_{k}$ represents the systematic uncertainties.

\section{Stochastic And Systematic UnCERTAinties}

In this section, we analyze both the process dynamics and the observation model in order to identify the sources of both types of uncertainties. The systematic uncertainties are thereby described by their lower and upper bounds.

\section{A. Uncertainties of Process Dynamics}

The stochastic and systematic uncertainties of the process model are handled in this section. In addition to the identification of the sources of both types of uncertainties, we derive their influence on the predicted state.

There exist three sources of systematic uncertainties in the process dynamics: initialization errors $\Delta \underline{g}_{k}$, discretization errors $\Delta \underline{m}_{k}$, and offsets $\Delta \underline{u}_{k}$ of the input signal. The initialization of the heart surface model can introduce systematic errors $\Delta \underline{g}_{k}$. As in [8] and [10], the position of the approximation points is based on measurements that can be corrupted by systematic uncertainties. These uncertainties then influence the calculation of $\mathbf{A}_{k}$ and $\mathbf{B}_{k}$. A derivation of lower and upper bounds for these errors will be given in Section IV-B. Furthermore, the process dynamics suffers from systematic disturbances $\Delta \underline{m}_{k}$, due to either unknown dynamics of the heart surface or discretization of its continuous behavior. In this paper, we define the errors in the process dynamics without taking the discontinuities and irregularities of the heart activity, e.g., extrasystoles, into account. The errors resulting from an insufficient description of the heart dynamics are hard to estimate and they strongly depend on the underlying model. For example, the physical models [8], [10] assume that uniformly distributed arterial pressure initiates the heart motion. Since the heart motion is caused by internal forces generated in a distributed fashion within the muscles, every point of the heart surface should be excited by a different force. This error appears linearly in the process model (1) and can be estimated by considering the error term $\mathbf{B}_{k} \Delta \underline{u}_{k}$ in the state-space model. The accuracy of a spatial and temporal discretization can, e.g., be estimated by means of Taylor series expansions. The error bounds of the spatial discretization given by a finite number of approximation 
functions, like radial basis or moving least square functions, are given in [20]. For temporal discretization, implicit or explicit methods can be applied with the error bounds estimated in [21]. Finally, a bias and drift of the input signal can lead to systematic errors in the state prediction. These errors are summarized by $\Delta \underline{u}_{k}$. Since the input signal is measured by a central line catheter, lower and upper bounds of the systematic errors should be defined in the data sheet of the catheter.

The stochastic uncertainties of the process dynamics are mainly determined by the noise of the input signal $\underline{u}_{k}$ and the uncertainty of the previous state.

In summary, the process dynamics model is affected by both stochastic and systematic uncertainties. The system equation that considers both types of uncertainties is described by

$$
\begin{aligned}
\underline{\boldsymbol{x}}_{k+1}= & \mathbf{A}_{k} \underline{\boldsymbol{x}}_{k}+\mathbf{B}_{k}\left(\hat{\hat{u}}_{k}+\underline{\boldsymbol{w}}_{k}\right)+ \\
& \underbrace{\mathbf{B}_{k} \Delta \underline{u}_{k}+\Delta \underline{\boldsymbol{x}}_{k+1}\left(\Delta \underline{g}_{k}\right)+\Delta \underline{m}_{k}}_{\underline{d}_{k}} .
\end{aligned}
$$

\section{B. Uncertainties of Observations}

In this section, we identify the sources of uncertainties in the observation model and then propagate them in order to determine their influence on the observations. The observation model is derived for the case when measurements are extracted from three camera views provided by a trinocular camera system.

The systematic uncertainties in the observation model $\Delta \underline{e}_{k}$ result from a limited validity of the measurement model or systematic calibration inaccuracies. These disturbances stem from initialization errors $\Delta \underline{s}_{k}$ and calibration errors $\Delta \underline{x}_{p}$ and $\Delta \underline{y} p$.

At first, the observation model is initialized with measurement data corrupted by systematic errors $\Delta \underline{s}_{k}$. They influence the calculation of the measurement matrix $\mathbf{H}_{k}$ and thus, cause biased measurements of the system state $\underline{y}_{k}\left(\Delta_{s_{k}}\right)$. Additionally, calibration errors contribute systematic uncertainties to the observation model. For example, a distortion leads to systematic correlated errors in the image position [22] and thus, in the 3D reconstruction. Also the uncertainties of calibration parameters lead to biased measurements [23]. In the following, the influence of these uncertainties on the reconstructed motion is analyzed.

The calibration accuracy is characterized by a pixel error that cannot be filtered out by calibration. These errors are given as reprojection errors after the calibration. Since they are different at every image point, especially in case of an incorrect distortion model, the accuracy of a 3D reconstruction depends on the position of a 3D point projection in the image frame. The reprojection errors can be estimated for every measurement point using common estimation approaches. However, this will lead to a high-dimensional nonlinear estimation problem impairing the real-time operability of the surgical system. We assume that the calibration error is bounded by $\Delta x_{p} \in[a, b]$ and $\Delta y_{p} \in[c, d]$ in both directions.

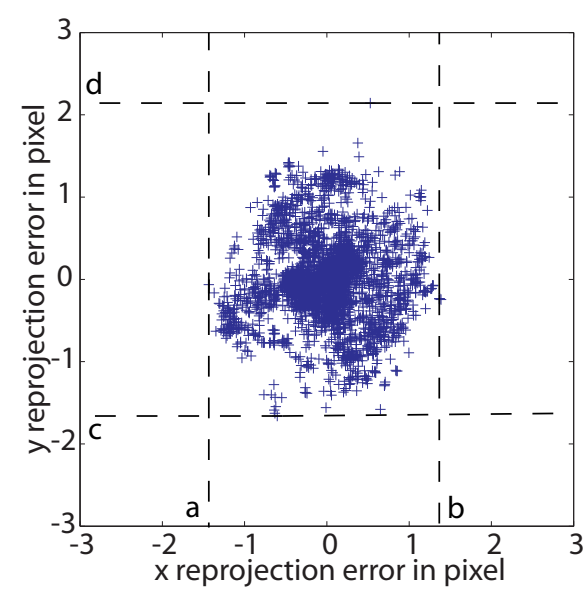

Fig. 2. Reprojection error provided by one camera after the calibration. The bounds of this pixel error are defined as maximal and minimal values in $\mathrm{x}$ and $\mathrm{y}$ direction. The calibration is performed by analyzing 50 images of a planar calibration board.

The bounds are determined by the minimum and maximum reprojection errors as shown in Fig. 2.

In general, the cameras are calibrated by using a linear pinhole camera model. The measurement $\underline{\hat{y}}_{k}$ described in equation (2) contains the 3D coordinates of a point $\underline{y}_{k}=$ $[x, y, z]^{T}$ in a world coordinate system. Now, a $3 \mathrm{D}$ point $\underline{y}_{k}=[x, y, z]^{T}$ would be projected to an image point with pixel coordinates $\underline{p}=\left[x_{p}, y_{p}\right]^{T}$ if there were no calibration errors. However, this image point shifts to the point $\underline{p}+\Delta p=\left[x_{p}+\Delta x_{p}, y_{p}+\Delta y_{p}\right]^{T}$ on the ideal image plane due to calibration inaccuracies, such as incomplete eliminated distortion or uncertainties of camera parameters. By combining these calibration errors in the vector $\Delta \underline{k}=$ $\left[\Delta x_{p}, \Delta y_{p}\right]^{T}$, we obtain the biased position of this point in camera coordinate system

$$
\underline{x}_{c}=\underline{\tilde{x}}_{c}+\Delta \underline{x}_{c}=\mathbf{K}^{-1} \underline{p}+\mathbf{J} \Delta \underline{k},
$$

where $\underline{\tilde{x}}_{c}$ represents the true position of this point in camera coordinate system and the Jacobian matrix $\mathbf{J}$ is given by

$$
\mathbf{J}=\frac{\partial \mathbf{K}^{-1} \underline{p}}{\partial \underline{k}} .
$$

The calibration matrix $\mathbf{K}$ contains the intrinsic camera parameters. The true image coordinates $p$ are represented as an augmented vector $\underline{p}=\left[x_{p}, y_{p}, 1\right]^{T}$. The camera coordinates $\underline{x}_{c}$ are normalized.

By using the matrix $\mathbf{M}$ containing the extrinsic camera parameters, we can express the transformation from a camera coordinate system into a world coordinate system in vectormatrix form

$$
\underline{x}_{c}=\mathbf{M} \underline{y}_{k},
$$

where a $3 \mathrm{D}$ point $\underline{y}_{k}=[x, y, z, 1]^{T}$ is represented as an augmented vector in homogeneous coordinates.

For the 3D reconstruction of a measurement point, we use the sets of correspondences $\underline{p}_{1}, \underline{p}_{2}, \underline{p}_{3}$ extracted from three camera views provided by a trinocular camera system. In this case, the augmented matrices $\mathbf{M}$ and $\mathbf{K}$ contain 
the extrinsic and intrinsic parameters of all three cameras. The components of the augmented vector $\underline{x}_{c}$ represent the position of measurement points in coordinate systems of three cameras. The vector $\underline{y}_{k}$ includes the possible reconstructions of measurement points in world coordinates. Then, the obtained overdetermined system (5) is minimized by using the quality criterion

$$
G(x)=\frac{1}{2}\left\|\underline{x}_{c}-\mathbf{M} \underline{y}_{k}\right\|^{2}=\left(\underline{x}_{c}-\mathbf{M} \underline{y}_{k}\right)^{\mathrm{T}}\left(\underline{x}_{c}-\mathbf{M} \underline{y}_{k}\right) \text {. }
$$

The necessary condition for a minimum

$$
\frac{\partial G\left(\underline{y}_{k}\right)}{\partial \underline{y}_{k}}=-\mathbf{M}^{\mathrm{T}} \underline{x}_{c}+\mathbf{M}^{\mathrm{T}} \mathbf{M} \underline{y}_{k}=0
$$

leads to normal equations with the analytic solution

$$
\underline{y}_{k}=\left(\mathbf{M}^{\mathrm{T}} \mathbf{M}\right)^{-1} \mathbf{M}^{\mathrm{T}} \underline{x}_{c} .
$$

Taking into account that the position of measurement points in camera coordinate system $\underline{x}_{c}$ is biased (4), we obtain the biased reconstruction

$$
\underline{y}_{k}=\left(\mathbf{M}^{\mathrm{T}} \mathbf{M}\right)^{-1} \mathbf{M}^{\mathrm{T}} \mathbf{K}^{-1} \underline{p}+\left(\mathbf{M}^{\mathrm{T}} \mathbf{M}\right)^{-1} \mathbf{M}^{\mathrm{T}} \mathbf{J} \Delta \underline{k}
$$

as a result of calibration errors.

The stochastic uncertainties of the observations can be determined experimentally by measuring stationary calibration objects.

In summary, the reconstruction of a $3 \mathrm{D}$ point is affected by both stochastic and systematic perturbations

$$
\begin{aligned}
\underline{y}_{k} & =\mathbf{H}_{k} \underline{\boldsymbol{x}}_{k}+\underline{\boldsymbol{v}}_{k}+\underline{e}_{k} \\
& =\left(\mathbf{M}^{\mathrm{T}} \mathbf{M}\right)^{-1} \mathbf{M}^{\mathrm{T}} \mathbf{K}^{-1} \underline{p}+\underline{\boldsymbol{v}}_{k}+\underline{e}_{k} .
\end{aligned}
$$

The vector $\underline{e}_{k}$ represents the systematic uncertainties

$$
\underline{e}_{k}=\underline{y}_{k}\left(\Delta \underline{s}_{k}\right)+\left(\mathbf{M}^{\mathrm{T}} \mathbf{M}\right)^{-1} \mathbf{M}^{\mathrm{T}} \mathbf{J} \Delta \underline{k} .
$$

\section{Estimation CONSIDERING STOCHASTIC AND SYSTEMATIC UNCERTAINTIES}

The most common way of treating uncertainties consists of modeling them as random quantities. Even though the characteristics of the disturbances are not precisely known, it is often attempted to impose a probability distribution on them. This can result in inconsistent state estimates or complicated models. For example, the considered linear models (3) and (6) would become nonlinear, if the system state is augmented by the unknown quantities. Besides stochastic approaches, set-valued representations [24], [25] of uncertain quantities have been evolved. Here, perturbations are characterized by their membership to a certain bounded set, which implies that they have to be amplitude-limited and that there is no weighting among the elements of a set. Thus, one cannot state that one element is "more probable" than another. In the following, stochastic and set-membership concepts will be combined.

A simultaneous treatment of stochastic and unknown but bounded uncertainties can be achieved by generalizing classical probability theory. The key idea is to use imprecise probabilities [26] instead of unique probabilities in order to characterize uncertain quantities. For this purpose, sets of densities will be employed in this paper, as it is described in [27], [28], and [29]. In the previous section, stochastic and systematic uncertainties have been identified. According to equation (1), the system state

$$
\underline{\boldsymbol{x}}_{k}=\underline{\hat{x}}_{k}+\underline{\boldsymbol{w}}_{k}+\underline{d}_{k}
$$

is affected additively by a Gaussian random noise $\underline{\boldsymbol{w}}_{k} \sim$ $\mathcal{N}\left(\underline{0}, \mathbf{C}_{k}\right)$ with zero-mean and covariance matrix $\mathbf{C}_{k} \in$ $\mathbb{R}^{n \times n}$ and by an unknown but bounded quantity $\underline{d}_{k} \in$ $\mathcal{D}_{k} \subset \mathbb{R}^{n}$. In the following, it is assumed that $\underline{\boldsymbol{w}}_{k}$ and $\underline{d}_{k}$ are independent. For a fixed $\underline{d}_{k}$, the uncertain system state $\underline{\boldsymbol{x}}_{k}$ is normally distributed with $\mathcal{N}\left(\underline{\hat{x}}_{k}+\underline{d}_{k}, \mathbf{C}_{k}\right)$. Since $\underline{d}_{k}$ is unknown, we cannot specify a unique distribution. But rather, continuing the line of thought, we have to consider the entire set

$$
\left\{\mathcal{N}\left(\underline{\hat{x}}_{k}+\underline{d}_{k}, \mathbf{C}_{k}\right) \mid \underline{d}_{k} \in \mathcal{D}_{k}\right\}
$$

of possible distributions for the system state. Obviously, $\underline{d}_{k}$ only affects the mean value, so the system state is characterized by a set of translated Gaussian densities with the same covariance matrix. Consequently, this set can be parameterized by a set of means $\mathcal{X}_{k}=\left\{\underline{\hat{x}}_{k}+\underline{d}_{k} \mid \underline{d}_{k} \in \mathcal{D}_{k}\right\}$ and a covariance matrix $\mathbf{C}_{k}$. According to these preparatory considerations, we can now use the Kalman filtering scheme to predict and update the system state.

\section{A. Prediction}

The estimated system state given by the set $\mathcal{X}_{k}^{\mathrm{e}}$ of means and the covariance matrix $\mathbf{C}_{k}^{\mathrm{e}}$ can be propagated by applying equation (1) to the expected values, i.e.,

$$
\underline{\hat{x}}_{k+1}^{\mathrm{p}}=\mathbf{A}_{k} \underline{\hat{x}}_{k}^{\mathrm{e}}+\mathbf{B}_{k} \underline{\hat{x}}_{k}+\underline{d}_{k}, \forall \underline{\hat{x}}_{k}^{\mathrm{e}} \in \mathcal{X}_{k}^{\mathrm{e}}, \underline{d}_{k} \in \mathcal{D}_{k} .
$$

This elementwise addition can also be written as the Minkowski sum

$$
\mathcal{X}_{k+1}^{\mathrm{p}}=\mathbf{A}_{k} \mathcal{X}_{k}^{\mathrm{p}} \oplus \mathcal{U}_{k}
$$

with

$$
\mathcal{U}_{k}=\left\{\mathbf{B}_{k} \underline{\hat{u}}_{k}+\underline{d}_{k} \mid \underline{d}_{k} \in \mathcal{D}_{k}\right\} .
$$

The predicted covariance matrix is computed from the wellknown Kalman filter equation

$$
\mathbf{C}_{k+1}^{\mathrm{p}}=\mathbf{A}_{k} \mathbf{C}_{k}^{\mathrm{p}} \mathbf{A}_{k}^{\mathrm{T}}+\mathbf{B}_{k} \mathbf{C}_{k}^{w} \mathbf{B}_{k}^{\mathrm{T}}
$$

Confining ourselves to linear models with Gaussian noise provides the advantage that the stochastic and systematic uncertainties remain distinguishable from each other. The influence of systematic perturbations to the system state at time step $k+1$ is represented by the set of possible means (8) and the stochastic uncertainty is characterized by the covariance matrix (9). In order to calculate equation (8) efficiently, ellipsoidal sets proved to be suitable representations for the systematic errors. An ellipsoid

$$
\mathcal{E}(\underline{\hat{c}}, \mathbf{X}):=\left\{\underline{x} \in \mathbb{R}^{n} \mid(\underline{x}-\underline{\hat{c}})^{\mathrm{T}} \mathbf{X}^{-1}(\underline{x}-\underline{\hat{c}}) \leq 1\right\}
$$


is defined by a midpoint $\underline{\hat{c}} \in \mathbb{R}^{n}$ and a nonnegative definite shape matrix $\mathbf{X} \in \mathbb{R}^{n \times n}$. By this, affine transformations can easily be calculated by

$$
\mathbf{A} \mathcal{E}(\underline{\hat{c}}, \mathbf{X})+\underline{b}=\mathcal{E}\left(\mathbf{A} \underline{\hat{c}}+\underline{b}, \mathbf{A} \mathbf{X} \mathbf{A}^{\mathrm{T}}\right) .
$$

The Minkowski sum of two ellipsoid does not in general yield an ellipsoid, but it can easily be approximated by an enclosing ellipsoid

$$
\mathcal{E}\left(\underline{\hat{c}}_{1}+\underline{\hat{c}}_{2}, \mathbf{X}(p)\right) \supseteq \mathcal{E}\left(\underline{\hat{c}}_{1}, \mathbf{X}_{1}\right) \oplus \mathcal{E}\left(\underline{\hat{c}}_{2}, \mathbf{X}_{2}\right)
$$

with

$$
\mathbf{X}(p)=\left(1+p^{-1}\right) \mathbf{X}_{1}+(1+p) \mathbf{X}_{2}
$$

for any $p>0$, as described in [24]. For

$$
p=\frac{\operatorname{trace}\left(\mathbf{X}_{1}\right)^{\frac{1}{2}}}{\operatorname{trace}\left(\mathbf{X}_{2}\right)^{\frac{1}{2}}},
$$

this outer approximation has a minimal sum of squares of semiaxes, i.e., the trace of $\mathbf{X}(p)$ is minimal. By characterizing the unknown but bounded uncertainties by $\mathcal{D}_{k}=$ $\mathcal{E}\left(\underline{0}, \mathbf{D}_{k}\right)$ and assuming that the actual state is given by the ellipsoid $\mathcal{X}_{k}^{\mathrm{e}}=\mathcal{E}\left(\underline{\hat{c}}_{k}^{\mathrm{e}}, \mathbf{X}_{k}^{\mathrm{e}}\right)$, the elementwise addition (7) simplifies to

$$
\begin{aligned}
\mathcal{X}_{k+1}^{\mathrm{p}} & \stackrel{\text { eq. (8) }}{=} \mathbf{A}_{k} \mathcal{E}\left(\underline{\hat{c}}_{k}^{\mathrm{e}}, \mathbf{X}_{k}^{\mathrm{e}}\right) \oplus\left(\mathbf{B}_{k} \underline{\hat{u}}_{k}+\mathcal{E}\left(\underline{0}, \mathbf{D}_{k}\right)\right) \\
& \stackrel{\text { eq. (10) }}{=} \mathcal{E}\left(\mathbf{A}_{k} \underline{\hat{c}}_{k}^{\mathrm{e}}, \mathbf{A}_{k} \mathbf{X}_{k}^{\mathrm{e}} \mathbf{A}_{k}^{\mathrm{T}}\right) \oplus \mathcal{E}\left(\mathbf{B}_{k} \underline{\hat{u}}_{k}, \mathbf{D}_{k}\right) \\
& \stackrel{\text { eq. (11) }}{\subseteq} \mathcal{E}\left(\underline{\hat{c}}_{k+1}^{\mathrm{p}}, \mathbf{X}_{k+1}^{\mathrm{p}}\right) .
\end{aligned}
$$

Finally, we have extended the Kalman prediction step to sets of Gaussian densities, where the predicted estimate is given by the set $\mathcal{E}\left(\underline{\hat{c}}_{k+1}^{\mathrm{p}}, \mathbf{X}_{k+1}^{\mathrm{p}}\right)$ of expected values and the covariance matrix $\mathbf{C}_{k+1}^{\mathrm{p}}$.

\section{B. Filtering}

The filtering step can be derived analogously. The unknown, but bounded bias $\underline{e}_{k}$ in equation (2) is affecting the measurement value $\underline{y}_{k}$, so we obtain a set

$$
\mathcal{Y}_{k}=\left\{\underline{\hat{y}}_{k}-\underline{e}_{k} \mid \underline{e}_{k} \in \mathcal{E}_{k}\right\}
$$

of possible measurements. For an ellipsoidal representation $\underline{e}_{k} \in \mathcal{E}_{k}=\mathcal{E}\left(\underline{0}, \mathbf{E}_{k}\right)$, this set becomes $\mathcal{Y}_{k}=\mathcal{E}\left(\underline{y}_{k}, \mathbf{E}_{k}\right)$. Every $\underline{\tilde{y}}_{k} \in \mathcal{Y}_{k}$ can then be processed elementwise with every prior or predicted mean $\underline{\hat{x}}_{k}^{\mathrm{p}} \in \mathcal{E}\left(\underline{\hat{c}}_{k}^{\mathrm{p}}, \mathbf{X}_{k}^{\mathrm{p}}\right)$ through the Kalman filtering equation

$$
\underline{\hat{x}}_{k}^{\mathrm{e}}=\left(\mathbf{I}-\mathbf{K}_{k} \mathbf{H}_{k}\right) \underline{\hat{x}}_{k}^{\mathrm{p}}+\mathbf{K}_{k} \underline{\tilde{y}}_{k},
$$

where

$$
\mathbf{K}_{k}=\mathbf{C}_{k}^{\mathrm{p}} \mathbf{H}_{k}^{\mathrm{T}}\left(\mathbf{C}_{k}^{v}+\mathbf{H}_{k} \mathbf{C}_{k}^{\mathrm{p}} \mathbf{H}_{k}^{\mathrm{T}}\right)^{-1}
$$

is the Kalman gain. The set of estimated means is obtained from the Minkowski sum and outer approximation

$$
\begin{aligned}
\mathcal{X}_{k}^{\mathrm{e}} \stackrel{\text { eq. (13) }}{=}\left(\mathbf{I}-\mathbf{K}_{k} \mathbf{H}_{k}\right) \mathcal{E}\left(\underline{\hat{c}}_{k}^{\mathrm{p}}, \mathbf{X}_{k}^{\mathrm{p}}\right) \oplus \mathbf{K}_{k} \mathcal{E}\left(\underline{\hat{y}}_{k}, \mathbf{E}_{k}\right) \\
\stackrel{\text { eq. (10) }}{=} \mathcal{E}\left(\left(\mathbf{I}-\mathbf{K}_{k} \mathbf{H}_{k}\right) \underline{\hat{c}}_{k}^{\mathrm{p}},\left(\mathbf{I}-\mathbf{K}_{k} \mathbf{H}_{k}\right) \mathbf{X}_{k}^{\mathrm{p}}\left(\mathbf{I}-\mathbf{K}_{k} \mathbf{H}_{k}\right)^{\mathrm{T}}\right) \\
\quad \oplus \mathcal{E}\left(\underline{\hat{y}}_{k}, \mathbf{K}_{k} \mathbf{E}_{k} \mathbf{K}_{k}^{\mathrm{T}}\right) \\
\stackrel{\stackrel{\complement}{ }}{\stackrel{\text { eq. (11) }}{ }} \mathcal{E}\left(\underline{\hat{c}}_{k}^{\mathrm{e}}, \mathbf{X}_{k}^{\mathrm{e}}\right) .
\end{aligned}
$$

TABLE I

BOUNDS OF CALIBRATION ERRORS.

\begin{tabular}{l|l|l|l|l}
\hline & \multicolumn{2}{|l|}{ high resolution } & \multicolumn{2}{l}{ low resolution } \\
\hline camera & $\mathrm{x}$ in pixel & $\mathrm{y}$ in pixel & $\mathrm{x}$ in pixel & $\mathrm{y}$ in pixel \\
\hline camera 1 & {$[-1.31,1.67]$} & {$[-1.57,1.80]$} & {$[-2.24,2.99]$} & {$[-2.47,1.87]$} \\
camera 2 & {$[-1.43,1.38]$} & {$[-1.62,2.14]$} & {$[-1.75,2.06]$} & {$[-1.17,1.10]$} \\
camera 3 & {$[-1.91,1.70]$} & {$[-1.64,2.24]$} & {$[-1.04,1.28]$} & {$[-1.27,1.12]$} \\
\hline
\end{tabular}

So, the new midpoint is computed by the well-known Kalman filter equation

$$
\underline{\hat{c}}_{k}^{\mathrm{e}}=\left(\mathbf{I}-\mathbf{K}_{k} \mathbf{H}_{k}\right) \underline{\hat{c}}_{k}^{\mathrm{p}}+\mathbf{K}_{k} \underline{\hat{y}}_{k}
$$

and the new shape matrix is

$$
\begin{aligned}
\mathbf{X}_{k}^{\mathrm{e}} & =\left(1+p^{-1}\right)\left(\mathbf{I}-\mathbf{K}_{k} \mathbf{H}_{k}\right) \mathbf{X}_{k}^{\mathrm{p}}\left(\mathbf{I}-\mathbf{K}_{k} \mathbf{H}_{k}\right)^{\mathrm{T}} \\
& +(1+p) \mathbf{K}_{k} \mathbf{E}_{k} \mathbf{K}_{k}^{\mathrm{T}},
\end{aligned}
$$

where an optimal $p$ can be chosen as in equation (12). The covariance matrix is obtained according to the standard Kalman filter equation

$$
\mathbf{C}_{k}^{\mathrm{e}}=\mathbf{C}_{k}^{\mathrm{p}}-\mathbf{K}_{k} \mathbf{H}_{k} \mathbf{C}_{k}^{\mathrm{p}}
$$

Thus, the estimated state is finally given by a set of Gaussian densities with the set $\mathcal{E}\left(\underline{\hat{c}}_{k}^{\mathrm{e}}, \mathbf{X}_{k}^{\mathrm{e}}\right)$ of means and the covariance matrix $\mathbf{C}_{k}^{\mathrm{e}}$. The influences of systematic perturbations are characterized by the set of means and the stochastic uncertainties are described by the covariance matrix.

A detailed derivation of the presented concept can be found in [29], where nonlinear models and arbitrary densities are also considered.

\section{Evaluation}

The performance of the proposed approach is evaluated by estimating the state of a real system represented by a pressure-regulated artificial heart. After describing the experimental setup, we analyze the influence of the calibration accuracy and model precision on the estimates of the heart surface motion. For this, systematic and stochastic errors are handled by the proposed estimation approach. By comparing the estimated results with measurements of a significantly higher accuracy, we check the consistency of the obtained estimation result.

\section{A. Experimental Setup}

The motion of the artificial heart is comparable to the real heart deformations. According to the measurement experiment performed in [30], the stabilized heart moves $0.59 \mathrm{~mm}$ in the lateral plane ( $x$-y-direction) and $2.1 \mathrm{~mm}$ out-of-plane ( $z$-direction). The motion of the artificial heart is reconstructed based on three camera views provided by a trinocular camera system. The Pike F-210 cameras [31] are placed at a distance of $0.5 \mathrm{~m}$ from the artificial heart, their focal length is about $35 \mathrm{~mm}$, and the field of view is $12.8 \mathrm{~cm} \times 17.02 \mathrm{~cm}$. The $1920 \times 1080$ pixel resolution allows for a highly accurate $3 \mathrm{D}$ reconstruction. The cameras are calibrated with a planar calibration board by using the camera calibration toolbox for MATLAB ${ }^{\mathrm{TM}}$. The toolbox 


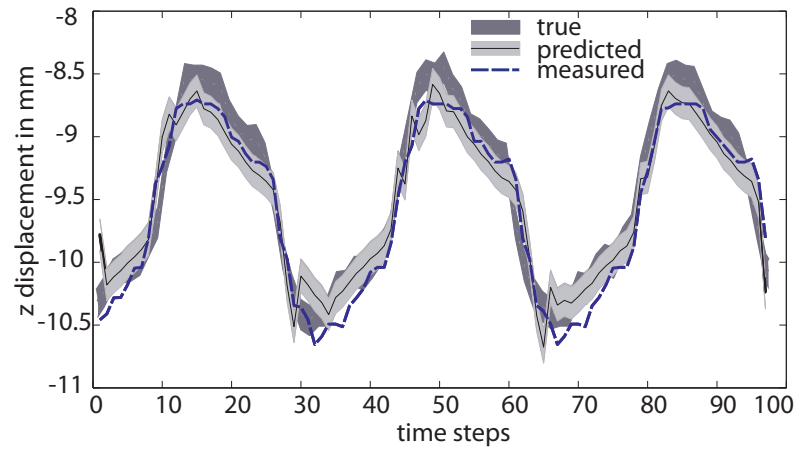

(a) One-step prediction considering only stochastic uncertainties. The ground truth (darkly shaded) is outside the $3 \sigma$ bounds (lightly shaded).

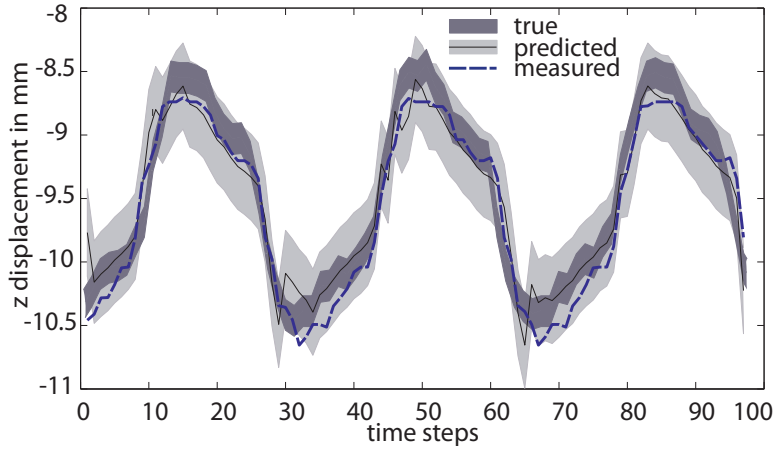

(b) One-step prediction considering stochastic and systematic uncertainties. The ground truth (darkly shaded) is inside the safety bounds (lightly shaded).

Fig. 3. Prediction of the heart surface motion and filtering with a single measurement at each time step. The ground truth, provided by the cameras with high resolution, is inside the safety bounds. Estimation without consideration of unknown uncertainties degrades the accuracy of the prediction.

provides the distribution of the reprojection error for every camera. By extrinsic calibration, the coordinate system of the first camera is taken as a world coordinate system. The coordinate systems of the other two cameras are transformed into the coordinate system of the first camera. In order to generate the ground truth, we measure the heart surface motion with the camera system at full resolution. This allows for achieving accurate measurements and thus leads to an accurate 3D reconstruction. Furthermore, the resolution is reduced to the lowest possible resolution of the cameras $960 \times 538$ pixel. In doing so, we try to approach the resolution of an endoscope, which is commonly $768 \times 576$ pixel. In comparison to endoscope, the proposed camera setup is characterized by large camera baselines. The endoscope is supposed to provide the measurements of the same accuracy if it is arranged in a small distance from the heart surface.

For evaluation, we use the process model proposed in [10]. This model is based on a physical description of the heart surface motion and employs spatial and temporal discretization methods that lead to systematic errors. The bounds of these errors are computed using methods proposed in [20], [21]. They are in the interval $[-1.1 \mu \mathrm{m}, 1.1 \mu \mathrm{m}]$. Additionally, we consider systematic errors stemming from the initialization of the process model. Since the measurements used for intialization are corrupted by bounded systematic errors, these errors influence the system state. These errors are assumed to be in the interval of the measurement system accuracy $[-0.2 \mathrm{~mm}, 0.2 \mathrm{~mm}]$. The input signal of the process dynamics model is given by the pressure inside the artificial heart. It is measured by a pressure sensor. In a clinical environment, the central line catheter can be applied. According to the data sheet of the sensor, it is calibrated for a measurement range of $100 \mathrm{hPa}$. The systematic disturbances of the input signal result from the sensor bias (about $0.375 \mathrm{hPa}$ ), drift, and nonlinearity $( \pm 0.2 \%$ of full scale or about $0.2 \mathrm{hPa}$ ).

Then, the systematic errors of the observation model are estimated. The systematic errors, not eliminated by calibration of the cameras, are determined by an lower and upper bound of the reprojection errors as described in Section IV-B. The bounds of these errors are given in
Table I. For comparison, the calibration results of the camera system with full and low resolution are also provided. The reprojection errors are of the same order for both types of calibrations. However, the reprojection errors of the camera system with lower resolution lead to higher errors in a world coordinate system because of the pixel size.

\section{B. Experimental Results}

To validate the proposed estimation approach, we predict the heart surface motion considering stochastic and unknown but bounded systematic errors of the process dynamics and observations. For the estimation, we employ the observations obtained by the cameras with lower resolution. The observations provided by the 3D reconstruction from full resolution images $1920 \times 1080$ pixel are taken as a ground truth. The heart surface motion in all directions is predicted. Due to a lack of space, only the prediction results of heart motion in $z$-direction are presented. As depicted in Fig. 3, the ground truth lies inside the estimated safety bounds. This means that when synchronizing surgical instruments with the heart surface motion, the safety bounds can prevent the robot from harming soft tissue. These dynamic bounds allow for evaluating the quality of the estimation at every time step. In particular, the actual achievable accuracy of the used measurement system can be determined online. If the bounds exceed the accuracy necessary for beating heart operations, more accurate measurements should be provided if the surgical instruments penetrate this region. For example, a force sensor can be employed to increase the accuracy at the intervention point. Inside the safety bounds, the robotic motion should be constrained. We believe that this can significantly contribute to the safety of the robotic operation and increase the robustness and reliability of the robotic surgery system.

\section{CONCLUSIONS}

The proposed approach for estimating the heart surface motion aims at improving the safety of robotic surgery on the beating heart. Model imprecisions and measurement inaccuracies lead to stochastic and systematic uncertainties. Neglecting them degrades the accuracy of the estimation. 
In this case, an operating robot can harm the soft heart tissue. However, existing approaches for the estimation of the heart surface motion are either deterministic or cope only with stochastic errors. In this paper, we propose an approach for estimating the heart surface motion that can cope with both stochastic and systematic uncertainties in the process dynamics and observations. It generates error bounds that can be used as safety bounds in a robotic control or as virtual fixtures. Inside these bounds, the robotic motion should be restricted in order to prevent the robot from accidentally harming the heart tissue. The main advantage of our concept is that the dynamic bounds, computed at every time step, are insensitive to occlusions. Furthermore, they allow for online assessing the quality of the estimation. The generation of these bounds should improve the safety and reliability of the robotic operation and reduce mental stress of the surgeon by restricting the robot motion in this area.

In future work, we plan to combine the estimated systematic error bounds with robot control mechanisms. Furthermore, the performance of the proposed concept will be evaluated in in-vivo experiments.

\section{ACKNOWLEDGMENTS}

This work was partially supported by the German Research Foundation (DFG) within the Research Training Groups RTG 1126 “Intelligent Surgery - Development of new computer-based methods for the future working environment in visceral surgery" and RTG 1194 "Self-organizing SensorActuator-Networks"

\section{REFERENCES}

[1] M. C. Cavusoglu, J. Rotella, W. S. Newman, S. Choi, J. Ustin, and S. S. Sastry, "Control Algorithms for Active Relative Motion Cancelling for Robotic Assisted Off-Pump Coronary Artery Bypass Graft Surgery," International Conference on Advanced Robotics, pp. 431-436, 2005.

[2] R. Ginhoux, J. Gangloff, M. de Mathelin, L. Soler, M. M. A. Sanchez, and J. Marescaux, "Active Filtering of Physiological Motion in Robotized Surgery Using Predictive Control," IEEE Transactions of Robotics, vol. 21, pp. 67-79, 2005.

[3] T. Ortmaier, M. Groeger, D. H. Boehm, V. Falk, and G. Hirzinger, "Motion Estimation in Beating Heart Surgery," IEEE Transactions on Biomedical Engineering, vol. 52, pp. 1729-1740, 2005.

[4] S. G. Yuen, D. T. Kettler, P. M. Novotny, R. D. Plowes, and R. D. Howe, "Robotic Motion Compensation for Beating Heart Intracardiac Surgery," International Journal of Robotics Research, pp. 1-18, 2009.

[5] M. Sauvée, P. Poignet, J. Triboulet, E. Dombre, E. Malis, and R. Demaria, "3D Heart Motion Estimation Using Endoscopic Monocular Vision System," Modeling and Control in Biomedical Systems, vol. 6, pp. 1-6, 2006.

[6] L. Cuvillon, J. Gangloff, M. de Mathelin, and A. Forgione, "Toward Robotized Beating Heart TECABG: Assessment of the Heart Dynamics Using High-Speed Vision," International conference on Medical Image Computing and Computer-Assisted Intervention, pp. 551-558, 2005.

[7] L. Chiari, U. D. Croce, A. Leardini, and A. Cappozzo, "Human Movement Analysis Using Stereophotogrammetry. Part 2: Instrumental Errors," Gait and Posture, vol. 21, pp. 197-211, 2005.

[8] T. Bader, A. Wiedemann, K. Roberts, and U. D. Hanebeck, "Modelbased Motion Estimation of Elastic Surfaces for Minimally Invasive Cardiac Surgery," in Proceedings of the 2007 IEEE International Conference on Robotics and Automation (ICRA 2007), Rome, Italy, Apr. 2007, pp. 2261-2266.
[9] S. G. Yuen, P. M. Novotny, and R. D. Howe, "Quasiperiodic Predictive Filtering for Robot-Assisted Beating Heart Surgery," IEEE International Conference on Robotics and Automation, pp. 3875-3880, 2008.

[10] E. Bogatyrenko, U. D. Hanebeck, and G. Szabo, "Heart Surface Motion Estimation Framework for Robotic Surgery Employing Meshless Methods," in Proceedings of the 2009 IEEE/RSJ International Conference on Intelligent Robots and Systems (IROS 2009), October 2009.

[11] R. Richa, A. P. L. Bó, and P. Poignet, "Beating Heart Motion Prediction for Robust Visual Tracking," IEEE International Conference on Robotics and Automation, pp. 1-6, 2010.

[12] G. Welch and G. Bishop, "An Introduction to the Kalman Filter," UNC-Chapel Hill, pp. 95-041, 2006.

[13] J. R. Huddle and D. A. Wismer, "Degradation of Linear Filter Performance Due to Modeling Error," IEEE Transactions Automatic Control, vol. 13, pp. 421-423, 1968.

[14] B. Fei, W. S. Ng, and C. K. Kwoh, "The Hazard Identification and Safety Insurance Control for Medical Robot," Proceedings of the EMBS International Conference, pp. 3022-3026, 2000.

[15] R. D. H. Shinsuk Park and and D. F. Torchiana, "Virtual Fixtures for Robotic Cardiac Surgery," Lecture Notes In Computer Science, vol. 2208, pp. 1419-1420, 2001.

[16] A. Bettini, P. Marayong, S. Lang, A. M. Okamura, and G. D. Hager, "Vision-Assisted Control for Manipulation using Virtual Fixtures," IEEE Transactions Robotic, vol. 20, pp. 953-966, 2004.

[17] J. Ren, R. V. Patel, K. A. McIsaac, G. Guiraudon, and T. M. Peters, "Dynamic 3-D Virtual Fixtures for Minimally Invasive Beating Heart Procedures," IEEE Transactions on Medical Imaging, vol. 27, pp. 1061-1070, 2008.

[18] H. Liu and P. Shi, "Meshfree Representation and Computation: Applications to Cardiac Motion Analysis," International Conference on Information Processing in Medical Imaging, pp. 560-572, 2003.

[19] R. Zahorec and M. Holoman, "Transatrial access for left atrial pressure monitoring in cardiac surgery patients," European Journal of Cardiothoracic Surgery, vol. 11, pp. 379-380, 1997.

[20] G. E. Fasshauer, Meshfree Approximation Methods with Matlab. Interdisciplinary Mathematical Sciences - Vol. 6, 2007.

[21] W. E. Boyce and R. C. DiPrima, Elementary Differential Equations and Boundary Value Problems. John Wiley and Sons, Inc., 2009.

[22] G. Florou and R. Mohr, "What Accuracy for 3D Measurements with Cameras?" International Conference on Pattern Recognition, pp. 354358, 1996.

[23] M. Zucchelli and J. Kosécka, "Motion Bias and Structure Distortion Induced by Calibration Errors," British Machine Vision Conference, pp. 663-673, 2001.

[24] A. Kurzhanski and I. Vályi, Ellipsoidal Calculus for Estimation and Control. Birkhäuser, 1997.

[25] F. C. Schweppe, Uncertain dynamic systems. Prentice-Hall, 1973.

[26] P. Walley, Statistical Reasoning with Imprecise Probabilities. London: Chapman and Hall, 1991, vol. Monographs on Statistics and Applied Probability 42.

[27] B. Noack, V. Klumpp, D. Brunn, and U. D. Hanebeck, "Nonlinear Bayesian Estimation with Convex Sets of Probability Densities," in Proceedings of the 11th International Conference on Information Fusion (Fusion 2008), Cologne, Germany, Jul. 2008, pp. 1-8.

[28] D. R. Morrell and W. C. Stirling, "Set-Valued Filtering and Smoothing," IEEE Transactions on systems, man and cybernetics, vol. 21, no. 1, pp. 184-193, Jan. 1991.

[29] B. Noack, V. Klumpp, and U. D. Hanebeck, "State Estimation with Sets of Densities considering Stochastic and Systematic Errors," in Proceedings of the 12th International Conference on Information Fusion (Fusion 2009), Seattle, Washington, July 2009.

[30] P. Cattina, H. Daveb, J. Gruenenfelder, G. Szekelya, M. Turin, and G. Zuend, "Trajectory of Coronary Motion and its Significance in Robotic Motion Cancellation," European Journal of Cardio-thoracic Surgery, vol. 25, pp. 786-790, 2004.

[31] PIKE Technical Manual V4.2.0, Allied Vision Technologies GmbH, September 2009. 